\title{
Structure and transitions of nucleon excitations via parity-expanded variational analysis
}

\author{
Finn M. Stokes ${ }^{* a b}$, Waseem Kamleh ${ }^{a}$ and Derek B. Leinweber ${ }^{a}$ \\ ${ }^{a}$ Special Research Centre for the Subatomic Structure of Matter, \\ Department of Physics, University of Adelaide, South Australia 5005, Australia \\ ${ }^{b}$ Jülich Supercomputing Centre, Institute for Advanced Simulation, \\ Forschungszentrum Jülich, Jülich D-52425, Germany \\ E-mail: f.stokes@fz-juelich.de
}

\begin{abstract}
The recently-introduced Parity Expanded Variational Analysis (PEVA) technique allows for the isolation of baryon eigenstates on the lattice at finite momentum free from opposite-parity contamination. We find that this technique introduces a statistically significant correction in extractions of the electromagnetic form factors of the ground state nucleon. It also allows first extractions of the elastic and transition form factors of nucleon excitations on the lattice. We present the electromagnetic elastic form factors and helicity amplitudes of two odd-parity excitations of the nucleon. These results provide valuable insight into the structure of these states, and allow for a connection to be made to quark-model states in this energy region.
\end{abstract}

37th International Symposium on Lattice Field Theory - Lattice2019

16-22 June 2019

Wuhan, China

*Speaker. 


\section{Introduction}

In lattice QCD, instead of the unstable finite-width resonances of nature, we observe a tower of stable excitations. These eigenstates are associated with the physical resonances in a non-trivial manner. Understanding the structure of the states observed in Lattice QCD will enable predictions of the infinite-volume observables of nature via effective field theory techniques $[1,2]$ or an extension of the Lellouch-Lüscher formalism [3].

Investigating the structure of excited states in lattice QCD is recognised as an important frontier in the field. Progress has already been made in the meson sector $[4,5]$. Here we tackle the more challenging problem of calculating such quantities in the baryon sector.

By using local three-quark operators on the lattice, both the CSSM [6] and the Hadron Spectrum Collaboration (HSC) [7] observe two low-lying odd-parity states in the resonance regimes of the $N^{*}(1535)$ and $N^{*}(1650)$. In the following we summarise our recent results on the elastic form factors of the ground state nucleon [8], these two odd-parity states, and the lowest-lying even-parity state accessible through the same operators [9]. In addition, we present preliminary results on the transition form factors for the two odd-parity states. These results were made possible through the development of the PEVA technique [10].

\section{Parity Expanded Variational Analysis (PEVA)}

The process of extracting elastic from factors of baryonic excited states via the PEVA technique is presented in full in Ref [8]. We provide here a brief summary of this process and the generalisations required to handle transition matrix elements.

We begin with a basis of $n$ conventional spin-1/2 operators $\left\{\chi_{i}(x)\right\}$ that couple to the states of interest. Adopting the Pauli representation, we introduce the PEVA projector [10]

$$
\Gamma_{ \pm \boldsymbol{p}} \equiv \frac{1}{4}\left(\mathbb{I}+\gamma^{4}\right)\left(\mathbb{I} \pm i \gamma^{5} \gamma^{k} \widehat{\boldsymbol{p}}^{k}\right)
$$

and construct a set of basis operators

$$
\begin{aligned}
\chi_{ \pm \boldsymbol{p} i}(x) & \equiv \Gamma_{ \pm \boldsymbol{p}} \chi_{i}(x) \\
\chi_{ \pm \boldsymbol{p} i^{\prime}}(x) & \equiv \pm \Gamma_{ \pm \boldsymbol{p}} \gamma^{5} \chi_{i}(x) .
\end{aligned}
$$

We then seek an optimised set of operators $\phi_{ \pm p}^{\alpha}(x)$ that each couple strongly to a single energy eigenstate $\alpha$. These optimised operators are constructed as linear combinations of the basis operators by solving a generalised eigenvalue problem as detailed in Ref. [10].

We can then construct the eigenstate-projected two-point correlation function

$$
G(\boldsymbol{p} ; t ; \alpha) \equiv \operatorname{Tr}\left(\sum_{x} \mathrm{e}^{-i \boldsymbol{p} \cdot \boldsymbol{x}}\left\langle\Omega\left|\phi_{ \pm \boldsymbol{p}}^{\alpha}(x) \bar{\phi}_{ \pm \boldsymbol{p}}^{\alpha}(0)\right| \Omega\right\rangle\right)
$$

and the three point correlation functions

$$
\mathscr{G}_{ \pm}^{3}\left(j_{C I}^{\mu} ; \boldsymbol{p}^{\prime}, \boldsymbol{p} ; t_{2}, t_{1} ; \alpha \rightarrow \beta\right) \equiv \sum_{\boldsymbol{x}_{1}, \boldsymbol{x}_{2}} \mathrm{e}^{-i \boldsymbol{p}^{\prime} \cdot \boldsymbol{x}_{2}} \mathrm{e}^{i\left(\boldsymbol{p}^{\prime}-\boldsymbol{p}\right) \cdot \boldsymbol{x}_{1}}\left\langle\Omega\left|\phi_{ \pm \boldsymbol{p}^{\prime}}^{\beta}\left(x_{2}\right) j_{C I}^{\mu}\left(x_{1}\right) \bar{\phi}_{+\boldsymbol{p}}^{\alpha}(0)\right| \Omega\right\rangle,
$$


where $j_{C I}^{\mu}(x)$ is the $O(a)$-improved [11] conserved vector current $j_{C I}^{\mu}(x)$ used in Ref. [12], inserted with a three-momentum transfer $\boldsymbol{q}=\boldsymbol{p}^{\prime}-\boldsymbol{p}$.

For the elastic case, this choice of current gives the matrix element

$$
\begin{aligned}
& \left\langle\alpha ; p^{\prime} ; s^{\prime}\left|j_{C I}^{\mu}(0)\right| \alpha ; p ; s\right\rangle \\
& \quad=\sqrt{\frac{m^{\alpha}}{E^{\alpha}(\boldsymbol{p})}} \sqrt{\frac{m^{\alpha}}{E^{\alpha}\left(\boldsymbol{p}^{\prime}\right)}} \bar{u}^{\alpha}\left(p^{\prime}, s^{\prime}\right)\left(\gamma^{\mu} F_{1}\left(Q^{2}\right)-\frac{\sigma^{\mu v} q^{v}}{2 m^{\alpha}} F_{2}\left(Q^{2}\right)\right) u^{\alpha}(p, s),
\end{aligned}
$$

where $Q^{2}=\boldsymbol{q}^{2}-\left(E^{\alpha}\left(\boldsymbol{p}^{\prime}\right)-E^{\alpha}(\boldsymbol{p})\right)^{2}$, and $F_{1}\left(Q^{2}\right)$ and $F_{2}\left(Q^{2}\right)$ are the Dirac and Pauli form factors. The matrix element can be extracted by taking appropriate ratios of the three- and two-point correlation functions. The Sachs electromagnetic form factors

$$
G_{E}\left(Q^{2}\right) \equiv F_{1}\left(Q^{2}\right)-\frac{Q^{2}}{\left(2 m^{\alpha}\right)^{2}} F_{2}\left(Q^{2}\right) \text { and } G_{M}\left(Q^{2}\right) \equiv F_{1}\left(Q^{2}\right)+F_{2}\left(Q^{2}\right)
$$

can then be extracted by taking linear combinations of the matrix elements.

We now move on to the transition form factors. The relevant matrix element is [13]

$$
\begin{aligned}
& \left\langle\beta^{-} ; p^{\prime} ; s^{\prime}\left|j_{C I}^{\mu}(0)\right| \alpha^{+} ; p ; s\right\rangle=\sqrt{\frac{m^{\alpha}}{E^{\alpha}(\boldsymbol{p})}} \sqrt{\frac{m^{\beta}}{E^{\beta}\left(\boldsymbol{p}^{\prime}\right)}} \\
& \quad \times \bar{u}^{\beta}\left(p^{\prime}, s^{\prime}\right)\left(\left(\delta^{\mu v}-\frac{q^{\mu} q^{v}}{q^{2}}\right) \gamma^{v} \gamma^{5} F_{1}^{*}\left(Q^{2}\right)-\frac{\sigma^{\mu v} q^{v}}{m^{\beta}-m^{\alpha}} \gamma^{5} F_{2}^{*}\left(Q^{2}\right)\right) u^{\alpha}(p, s),
\end{aligned}
$$

where $F_{1}^{*}\left(Q^{2}\right)$ and $F_{2}^{*}\left(Q^{2}\right)$ are Dirac- and Pauli-like transition form factors. We can then take ratios and linear combinations to obtain the transverse helicity amplitude

$$
A_{1 / 2}\left(Q^{2}\right) \equiv 2 \sqrt{\frac{Q^{2}+\left(m^{\beta}-m^{\alpha}\right)^{2}}{8 m^{\alpha}\left(m^{\beta 2}-m^{\alpha 2}\right)}}\left(F_{1}^{*}\left(Q^{2}\right)+F_{2}^{*}\left(Q^{2}\right)\right) .
$$

\section{Ground state nucleon}

We study the extraction of the elastic form factors of the ground state nucleon in detail in Ref. [8]. This analysis is performed on the PACS-CS $(2+1)$-flavour full-QCD ensembles [14], made available through the ILDG [15]. In the paper we demonstrate the efficacy of variational analysis techniques in general, and PEVA specifically, at controlling excited-state contaminations in the electric form factor. Both the PEVA and conventional variational analysis show clear and clean plateaus, supporting previous work demonstrating the utility of variational analysis in calculating baryon matrix elements [16, 17].

Here we focus on the particular case of the magnetic form factor, where we found evidence that the conventional analysis is contaminated by opposite-parity states. In Fig. 1a we plot a comparison of magnetic form factor plateaus produced by a conventional variational analysis (using an initial basis of $n=8$ operators), with an equivalent extraction via the PEVA technique (with the basis parity-expanded to $2 n=16$ operators). We see a significant difference in the plateaus extracted by the two techniques for the singly represented quark sector. If we take the correlated ratio of the 


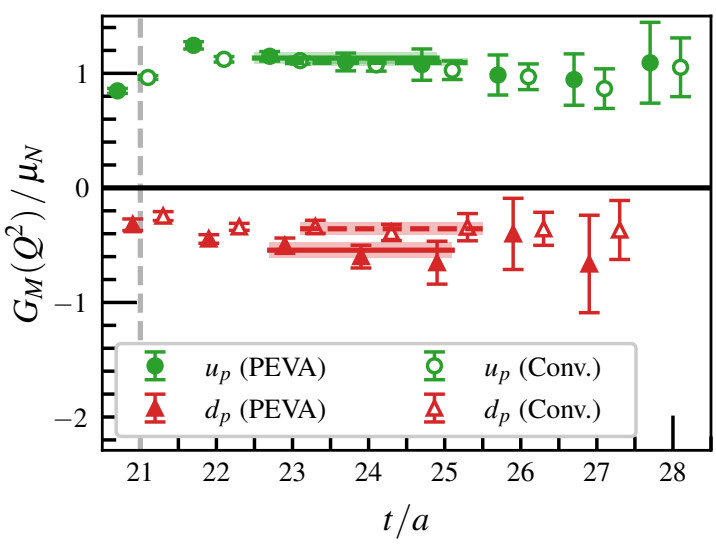

(a) Plateaus at $Q^{2}=0.166(4)$

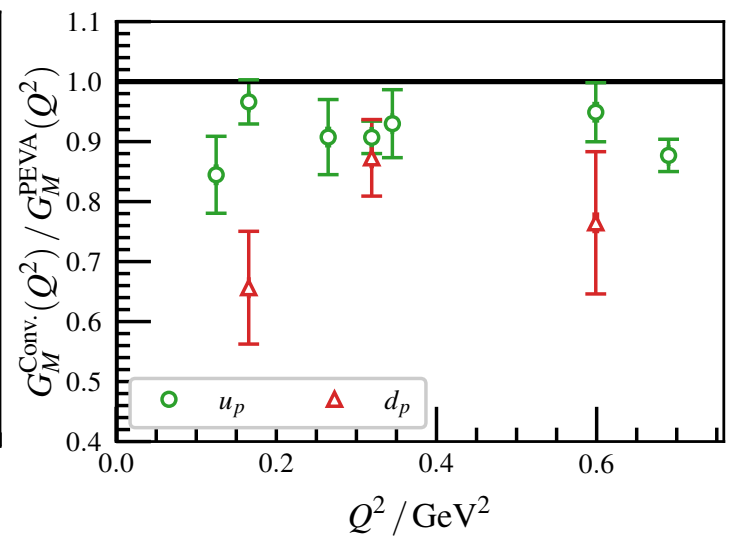

(b) Ratios of conventional plateaus to PEVA

Figure 1: Comparison of conventional and PEVA extractions of $G_{M}\left(Q^{2}\right)$ for the ground-state nucleon at $m_{\pi}=156 \mathrm{MeV}$. Results are contributions for single quarks of unit charge from the doubly represented quark sector $\left(u_{p}\right)$ and the singly represented quark sector $\left(d_{p}\right)$.

extracted values, as shown for a range of kinematics in Fig. 1b, we see a consistent underestimation of the value by the conventional analysis. This shows $\sim 20 \%$ underestimation of the magnitude of the contributions to the magnetic form factor from the singly represented quark flavour in the conventional analysis.

The difference between the two analyses is that the PEVA approach provides additional interpolator degrees of freedom to improve the ground state interpolating field at finite momentum. As such it is clear that the difference between the two extractions is from contaminating states that are present in the conventional analysis but removed by the parity expansion. As such, the PEVA technique is critical for precision measurements of nucleon form factors.

\section{Excitations}

With our local three-quark operators, we observe two low-lying odd-parity eigenstates in the resonance regimes of the $N^{*}(1535)$ and $N^{*}(1650)$. In Ref. [9], we investigate the elastic form factors of these states. There we find that opposite parity contaminations have a large effect on both the electric and magnetic form factors, and the PEVA technique is critical for even a qualitatively correct extraction.

We focus our investigation at heavier pion masses, where these lattice states lie below the relevant two-particle scattering thresholds on the finite volume. At these masses, we find that these states look remarkably similar to constituent quark model predictions for the $N^{*}(1535)$ and $N^{*}(1650)$.

We find the size of these lattice eigenstates to be similar to the ground state nucleons. As shown in Fig. 2, their magnetic moments agree well with constituent quark model predictions for the continuum states.

We also present here preliminary results for the transition form factors from the ground state to each of these two lattice eigenstates. These results will be presented in more detail in an upcoming 


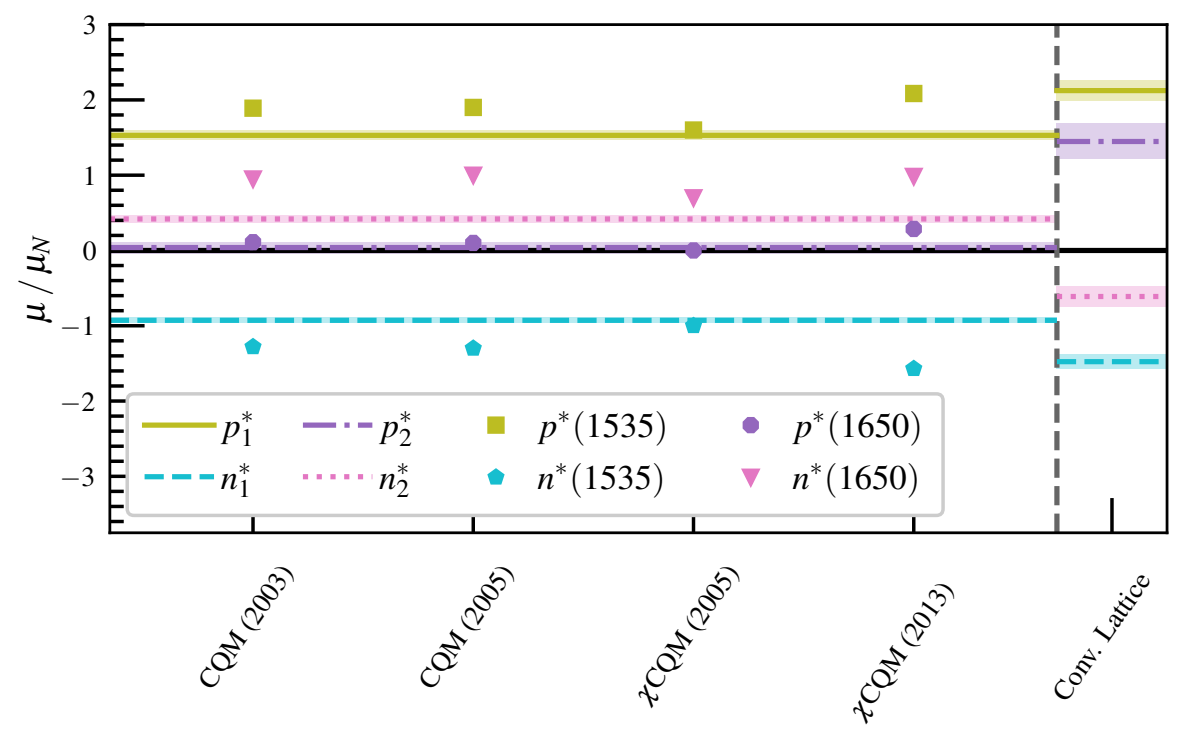

Figure 2: Comparison between lattice calculations of the magnetic moments of two odd-parity nucleon excitations at $m_{\pi}=702 \mathrm{MeV}$ and quark model predictions $[18,19,20]$ for the $N^{*}(1535)$ and $N^{*}(1650)$ resonances. The shaded bands on the left-hand side of the plot indicate the magnetic moments calculated via the PEVA technique in lattice QCD, and symbols denote the quark model predictions. Lattice calculations of the magnetic moments using conventional parity projection are plotted to the right of the vertical dashed line.

paper. In Fig. 3 we compare the ratio of the transverse helicity amplitudes of the proton and neutron to constituent quark model predictions. Taking this ratio allows us to cancel out some of the model dependence of the constituent quark model result. Similar to the magnetic moments above, we compare the first negative-parity lattice excitation $\left(N_{1}^{*}\right)$ to a quark model prediction for the $N^{*}(1535)$, and the second lattice excitation $\left(N_{2}^{*}\right)$ to a prediction for the $N^{*}(1650)$. We once again find good agreement between the structure of the lattice eigenstates at the heavier pion masses and the constituent quark model prediction.

We see strong agreement between the lattice eigenstates and constituent quark model predictions at these heavier pion masses. This suggests that while the dynamics are much more complicated at the physical point, and the constituent quark model alone does not appear to give a good description of these resonances in nature, as the pion mass increases the constituent quark model describes the excitations rather well. The agreement of the lighter state with the constituentquark-model $N^{*}(1535)$ is consistent with predictions from Hamiltonian Effective Field Theory (HEFT) [1]. However, the agreement of the heavier state with the constituent-quark-model $N^{*}(1650)$ suggests that future HEFT studies should explore the incorporation of two bare basis states associated with the two different localised states observed herein.

In Ref. [9], we also investigate the lowest-lying even-parity excitation of the nucleon observed on the lattice. We find that it has a charge radius approximately $30 \%$ larger than the ground state, and a remarkably similar magnetic moment to the ground state. This is consistent with the state being a radial excitation of the ground-state nucleon as seen in Refs. [22]. 


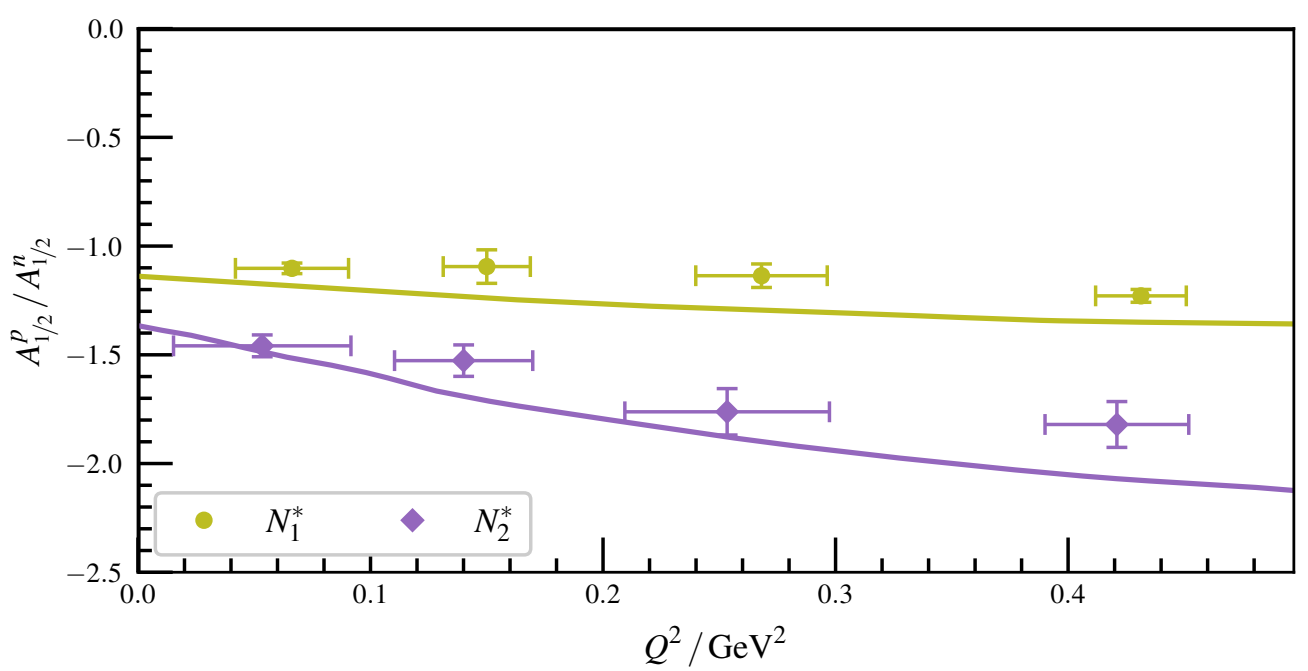

Figure 3: Constituent quark model predictions [21] (lines) and PEVA extractions (points) of the ratio of proton to neutron helicity amplitudes at $m_{\pi}=702 \mathrm{MeV}$.

\section{Conclusion}

The PEVA technique is critical to correctly extracting the form factors of nucleon excitations on the lattice. Such extractions give us insight into the structure of these finite-volume states. In addition, even for the ground state we found evidence that the conventional analysis was contaminated by opposite-parity states. For the kinematics considered here, we observe $\sim 20 \%$ underestimation of the contributions to the magnetic form factor from the singly represented quark flavour at lighter pion masses. All these results make it clear that the PEVA technique is critical for precision measurements of nucleon form factors and for any study of the structure of nucleon excitations.

\section{Acknowledgements}

This research was undertaken with the assistance of resources from the Phoenix HPC service at the University of Adelaide, the National Computational Infrastructure (NCI), which is supported by the Australian Government, and by resources provided by the Pawsey Supercomputing Centre with funding from the Australian Government and the Government of Western Australia. These resources were provided through the National Computational Merit Allocation Scheme and the University of Adelaide partner share. This research is supported by the Australian Research Council (ARC) through grants no. DP140103067, DP150103164, LE160100051, and DP190102215.

\section{References}

[1] Z.-W. Liu, W. Kamleh, D. B. Leinweber, F. M. Stokes, A. W. Thomas and J.-J. Wu, Hamiltonian effective field theory study of the $\mathbf{N}^{*}(\mathbf{1 5 3 5})$ resonance in lattice QCD, Phys. Rev. Lett. 116 (2016) 082004 [arXiv:1512.00140].

[2] Z.-W. Liu, W. Kamleh, D. B. Leinweber, F. M. Stokes, A. W. Thomas and J.-J. Wu, Hamiltonian effective field theory study of the $\mathbf{N}^{*}(\mathbf{1 4 4 0})$ resonance in lattice QCD, Phys. Rev. D95 (2017) 034034. 
[3] L. Lellouch and M. Luscher, Weak transition matrix elements from finite volume correlation functions, Commun. Math. Phys. 219 (2001) 31 [hep-lat/0003023].

[4] B. J. Owen, W. Kamleh, D. B. Leinweber, M. S. Mahbub and B. J. Menadue, Transition of $\rho \rightarrow \pi \gamma$ in lattice QCD, Phys. Rev. D92 (2015) 034513 [arXiv: 1505 . 02876].

[5] R. A. Briceno, J. J. Dudek, R. G. Edwards, C. J. Shultz, C. E. Thomas and D. J. Wilson, The resonant $\pi^{+} \gamma \rightarrow \pi^{+} \pi^{0}$ amplitude from Quantum Chromodynamics, Phys. Rev. Lett. 115 (2015) 242001.

[6] M. S. Mahbub, W. Kamleh, D. B. Leinweber, P. J. Moran and A. G. Williams, Low-lying Odd-parity States of the Nucleon in Lattice QCD, Phys. Rev. D87 (2013) 011501(R) [arXiv: 1209.0240 ].

[7] HADRON SPECTRUM collaboration, Flavor structure of the excited baryon spectra from lattice QCD, Phys. Rev. D87 (2013) 054506 [arXiv:1212.5236].

[8] F. M. Stokes, W. Kamleh and D. B. Leinweber, Opposite-Parity Contaminations in Lattice Nucleon Form Factors, Phys. Rev. D99 (2019) 074506 [arXiv: 1809.11002 ].

[9] F. M. Stokes, W. Kamleh and D. B. Leinweber, Elastic Form Factors of Nucleon Excitations in Lattice QCD, arXiv:1907.00177.

[10] F. M. Stokes, W. Kamleh, D. B. Leinweber, M. S. Mahbub, B. J. Menadue and B. J. Owen, Parity-expanded variational analysis for nonzero momentum, Phys. Rev. D92 (2015) 114506.

[11] G. Martinelli, C. T. Sachrajda and A. Vladikas, A Study of 'improvement' in lattice QCD, Nucl. Phys. B358 (1991) 212.

[12] S. Boinepalli, D. B. Leinweber, A. G. Williams, J. M. Zanotti and J. B. Zhang, Precision electromagnetic structure of octet baryons in the chiral regime, Phys. Rev. D74 (2006) 093005.

[13] B. J. Owen, A variational approach to hadron structure in lattice QCD, Ph.D. thesis, Adelaide U., Sch. Chem. Phys., 2015.

[14] PACS-CS collaboration, 2+1 Flavor Lattice QCD toward the Physical Point, Phys. Rev. D79 (2009) 034503 [arXiv:0807.1661].

[15] M. G. Beckett, B. Joo, C. M. Maynard, D. Pleiter, O. Tatebe and T. Yoshie, Building the International Lattice Data Grid, Comput. Phys. Commun. 182 (2011) 1208 [arXiv: 0910 . 1692].

[16] J. Dragos, R. Horsley, W. Kamleh, D. B. Leinweber, Y. Nakamura, P. E. L. Rakow et al., Nucleon matrix elements using the variational method in lattice QCD, Phys. Rev. D94 (2016) 074505.

[17] B. J. Owen, J. Dragos, W. Kamleh, D. B. Leinweber, M. S. Mahbub, B. J. Menadue et al., Variational Approach to the Calculation of gA, Phys. Lett. B723 (2013) 217 [arXiv:1212.4668].

[18] W.-T. Chiang, S. N. Yang, M. Vanderhaeghen and D. Drechsel, Magnetic dipole moment of the $S_{11}(1535)$ from the $\gamma p \rightarrow \gamma \eta p$ reaction, Nucl. Phys. A723 (2003) 205 [nucl-th/0211061].

[19] J. Liu, J. He and Y. B. Dong, Magnetic moments of negative-parity low-lying nucleon resonances in quark models, Phys. Rev. D71 (2005) 094004.

[20] N. Sharma, A. Martinez Torres, K. P. Khemchandani and H. Dahiya, Magnetic moments of the low-lying 1/2- octet baryon resonances, Eur. Phys. J. A49 (2013) 11 [arXiv:1207.3311].

[21] S. Capstick and B. D. Keister, Baryon current matrix elements in a light front framework, Phys. Rev. D51 (1995) 3598 [nucl-th/9411016].

[22] D. S. Roberts, W. Kamleh and D. B. Leinweber, Wave Function of the Roper from Lattice QCD, Phys. Lett. B725 (2013) 164 [arXiv:1304.0325]. 